\title{
DO SIGH BREATHS AUGMENT PRESSURE SUPPORT VENTILATION (PSV) IN PRETERM
} INFANTS?

\author{
P. Mallya ${ }^{1}$, S. Gupta ${ }^{1}$, S. Sinha ${ }^{2}$ \\ ${ }^{l}$ Neonatology, University Hospital of North Tees, Stockton, ${ }^{2}$ Neonatology, James Cook University Hospital, \\ Middlesbrough, UK
}

Background: PSV is a patient triggered, pressure limited, flow cycled mode of ventilation. Synchrony during inspiration and expiration, and flow dynamics of PSV might facilitate weaning from ventilator. In preterm infants PSV usage has been limited because of unreliable respiratory drive.

Objective: To compare respiratory parameters between Full PSV $\left(\mathrm{PS}_{\max }\right)$ alone and $\mathrm{PS}_{\max }$ with sigh breaths of SIMV (SIMV+PSV ${ }_{\max }$ ) during weaning from mechanical ventilation.

Methods: Breath by breath pulmonary mechanics data was collected from each infant during two epochs of ventilation using a standardised protocol and random crossover scheme. A total of 10,913 breaths were analysed from 10 ventilated preterm infants $<32$ weeks gestation, recovering from RDS. Both forms of ventilation provided $5-8 \mathrm{mls} / \mathrm{kg}$ expired tidal volume $\left(\mathrm{VT}_{\mathrm{e}}\right)$. Two tailed t-test was used for analyses. Ethics approval and parental consent was sought.

\section{Results:}

\begin{tabular}{|l|l|l|}
\hline \multicolumn{1}{|l|}{} & SIMV/PSmax $(\mathrm{n}=5603)$ & PSmax $(\mathrm{n}=5310)$ \\
\hline Expired tidal vloume $(\mathrm{ml} / \mathrm{kg})+/-$ & $6.73+/-0.02$ & $6.64+/-0.04$ \\
\hline Inspired tidal volume $(\mathrm{ml} / \mathrm{kg})+/-$ & $6.66+/-0.02$ & $6.65+/-0.04$ \\
\hline $\begin{array}{l}\text { Total minute ventilation } \\
(\mathrm{m} 1 / \mathrm{kg} / \mathrm{min})+/-\end{array}$ & $391.6+/-1.1$ & $403.8+/-1.5 \mathrm{p}<0.05$ \\
\hline Total Respiratory rate $(\mathrm{bpm})$ & 58.7 & $60.8 \mathrm{p}<0.05$ \\
\hline $\begin{array}{l}\text { Peak Inspiratory Pressure }(\mathrm{cms} \\
\text { H2O) }\end{array}$ & $15.74+/-0.04$ & $15.61+/-0.04$ \\
\hline Mean airway pressure $(\mathrm{cms} \mathrm{H2O)}$ & $7.85+/-0.02$ & $7.79+/-0.02$ \\
\hline End expiratory pressure $(\mathrm{cms} \mathrm{H2O)}$ & $4.94+/-0.01$ & $4.97+/-0.01$ \\
\hline Fraction of inspired oxygen $(\%)$ & $21.47+/-0.02$ & $22.36+/-0.03 \mathrm{p}<0.05$ \\
\hline
\end{tabular}

[Respiratory parameters on SIMV/PSmax vs. PSmax]

Conclusions: Minute ventilation is preserved during PSV irrespective of SIMV sigh breaths during weaning. Addition of sigh breaths to PSV stabilizes breathing at comparable respiratory parameters. 